\title{
Determinantes de estereotipos sexistas en un estudio local cuantitativo en Guerrero, México
}

Determinants of sexist stereotypes in a quantitative local study in Guerrero, Mexico

Jorge Luis Triana Sánchez ${ }^{1}$

\section{Resumen}

En este artículo se discute el papel de algunos factores sociodemográficos sobre la incidencia de estereotipos sexistas en personas adultas del área urbana del municipio de Acapulco, en el estado de Guerrero, México. Con datos de una encuesta local, se estiman regresiones logísticas para examinar siete estereotipos relativos a la remuneración y posición de la mujer en el trabajo, el ejercicio de su sexualidad, el cuidado de los hijos, y las labores domésticas. Los resultados muestran que la presencia de estereotipos sexistas aumenta con la edad, pero disminuye en función de la escolaridad. Un hallazgo emergente es que no solamente el sexo de la persona encuestada determina la incidencia de algunos estereotipos sexistas, sino también el sexo de quien realiza la entrevista.

Palabras clave: género, estereotipos, variables sociales, sexismo, regresión logística

\section{Abstract}

This paper analyzes the effect of sociodemographic factors on the prevalence of sexist stereotypes in adults in the urban area of the municipality of Acapulco, in the state of Guerrero, Mexico. Logistic regressions are estimated using data from a local survey to examine seven stereotypes about the remuneration and position of women at work, the exercise of their sexuality, childcare and domestic chores. The results show that the prevalence of sexist stereotypes increases with age, but decreases with schooling; an emerging finding is that not only the sex of the person surveyed determines the prevalence of some sexist stereotypes, but also the sex of the person conducting the interview.

Keywords: gender, stereotypes, social variables, sexism, logistic regression

Recibido: 10 de junio de 2021 Aceptado: 20 de diciembre de 2021 Publicado: 7 de enero de 2022

${ }^{1}$ Doctor en Política Pública. Universidad de Ciencias y Artes de Chiapas (UNICACH), San Cristóbal de Las Casas, México. Correo electrónico: jorgetriana@outlook.com ID https://orcid.org/0000-0003-2036-6846. 


\section{Introducción}

El objetivo de este trabajo es analizar el efecto de diversos factores sociodemográficos sobre la incidencia de estereotipos sexistas entre habitantes del área urbana del municipio de Acapulco, Guerrero. El interés particular por el efecto de estas variables sociales sobre los estereotipos proviene de antecedentes en la literatura, que han postulado asociaciones analíticas entre la transformación de las relaciones de género y algunos cambios en materia social, demográfica, económica y cultural en México (Rojas, 2016). Algunos estudios destacan la importancia relacional entre las categorías etnia y género (Díaz-Cervantes, 2014; González y Viadero, 2010), mientras que otros enfatizan el papel del estado civil en el empoderamiento de las mujeres y la confrontación de estereotipos sexistas, en particular a partir de una disolución conyugal (Bonelli, 2017; Castañeda, 2007). Se ha encontrado además evidencia empírica correlacional -a un nivel bivariado- entre la prevalencia de estereotipos sexistas y características sociodemográficas como el sexo, educación, edad y estrato socioeconómico (Frías y Erviti, 2012; Triana, 2020).

Partiendo de lo anterior, se busca comprobar si dichas asociaciones analíticas y correlaciones empíricas se sostienen en un nivel explicativo. Para tal efecto, y desde un enfoque de investigación cuantitativo, se utilizan regresiones logísticas que permiten aislar el efecto marginal de cada variable sociodemográfica sobre la probabilidad de que una persona esté de acuerdo con alguno de los siguientes estereotipos: 1) los hombres deben ganar más salario que las mujeres; 2) los hombres deben ocupar mejores puestos que las mujeres en los trabajos; 3) las mujeres que trabajan descuidan a sus hijos; 4) las mujeres casadas deben tener relaciones sexuales con su esposo cuando él quiera; 5) las mujeres deben vestirse sin escotes para que no las molesten los hombres; 6) sólo las mujeres deben ser las responsables del cuidado de los hijos, y; 7) la naturaleza de la mujer la inclina a realizar las tareas domésticas.

Los estereotipos de género han sido ampliamente estudiados en términos históricos (Machillot, 2013), jurídicos (Cook \& Cusack, 2009), sociológicosantropológicos (Gutmann, 2000; Lamas, 1986; Martín, 2008), psicosociológicos (Ashmore et al, 1986; Glick \& Fiske, 1997), publicitarios (Delgado et al, 1998; Pearson et al, 1993) y multidisciplinarios (Basow, 1992). En el presente trabajo se abordan los estereotipos de género desde una visión sociológica, y en particular desde una perspectiva teórica feminista, en la cual el género se entiende como "la simbolización que cada cultura elabora sobre la diferencia sexual, estableciendo normas y expectativas sociales sobre los papeles, las conductas y los atributos de las personas a partir de sus cuerpos” (Lamas, 2002, p. 52), mientras que los roles de género constituyen un "conjunto de normas y prescripciones que dicta la sociedad y la cultura 
sobre el comportamiento femenino o masculino" (Lamas, 1986, p. 188). Estos roles son procesados a un nivel psicosocial como estereotipos que moldean las expectativas en razón de género, promoviendo la perpetuación de desigualdades sociales entre hombres y mujeres (Swim \& Hyers, 2009).

\section{Estereotipos, género y sexismo}

Los estereotipos son generalizaciones sociales que se componen de rasgos comúnmente asociados a cierto grupo con el fin de diferenciarlo de otros, como una especie de atajo mental para describir a sus miembros a través de una generalización extrema, que suele conducir a descripciones negativas e imprecisas de las personas (Stangor, 2009). Estas generalizaciones no son expresiones individuales aisladas, sino que se conforman colectivamente como "creencias fuertemente arraigadas acerca de las características que se atribuyen a ciertas categorías de personas" (Stern, 2007, p. 106).

Se considera a los estereotipos como una consecuencia inherente de la cognición humana, altamente sensible al contexto social en el cual éstos emergen, pero también a motivaciones personales. Se componen de creencias ambivalentes que reflejan las relaciones entre grupos, comúnmente sesgadas a las percepciones de comportamientos negativos y extremos que promueven la separación social grupal; sus contenidos son almacenados en estructuras cognitivas internas sujetas a las dinámicas de poder y el statu quo, por lo que se convierten en herramientas para preservar jerarquías sociales, racionalizar entornos de desigualdad y fomentar la hostilidad entre grupos (Operario \& Fiske, 2003).

Así, los estereotipos se posicionan como instrumentos eficientes para la configuración del orden social, pues al predisponer un determinado comportamiento en cierto grupo, tiende a provocar de manera inconsciente en los demás una respuesta esperada, reforzando a su vez el estereotipo en cuestión. Este mecanismo de reproducción y la consecuente normalización de los comportamientos provocados, explica su resistencia al cambio a pesar de la modificación de las condiciones sociales en las cuales se originaron (Stern, 2007).

Los estereotipos suelen activarse a través de categorías sociales como el sexo, edad, estado civil, clase social, apariencia, nivel educativo, situación laboral, religión, estatus profesional, etnia, estatus migratorio o nacionalidad; cuando se activan a través del género, se apela a "un conjunto estructurado de creencias y expectativas compartidas, dentro de una sociedad, acerca de las características que poseen (componente descriptivo) y deben poseer (componente prescriptivo) las mujeres y los hombres como grupos" (Pla et al, 2013, p. 22). Si bien los estereotipos de género capturan, en mayor o menor medida, las diferencias entre lo que piensan y hacen 
hombres y mujeres, también influyen en la manera en que los sujetos se definen a sí mismos y la manera en que son tratados por otros, prescribiendo en términos psicosociales la manera en que deberían ser y cómo deberían comportarse en distintos ámbitos de la vida (Ellemers, 2018).

Kite et al (2008) plantean que los estereotipos de género se construyen a partir de dos creencias centrales: que las mujeres se preocupan por el bienestar de otras personas, y que los hombres son asertivos y controladores. De estos dos postulados se derivan las creencias de que las mujeres suelen ser más emocionales, comprensivas, amables y devotas, mientras que los hombres suelen ser activos, seguros de sí mismos, independientes y competitivos; de ahí que los hombres se conviertan en líderes, proveedores y jefes del hogar, mientras que las mujeres ejerzan como cuidadoras, amas de casa y consejeras. Lo anterior debido a que se piensa que los hombres están particularmente dotados de una capacidad para el razonamiento abstracto y la resolución de problemas, mientras que las mujeres gozan de habilidades artísticas y razonamiento verbal. Finalmente, los estereotipos de género postulan también la apariencia de cada sexo: mientras que los hombres se ven fuertes, atléticos y agresivos, las mujeres se ven bonitas y delicadas.

La expectativa social del rol masculino también suele anclarse a su vinculación con lo público, ya que es el mundo exterior donde ejerce su papel de proveedor de las necesidades del hogar, mientras que la expectativa social de la función femenina se limita al ámbito doméstico, pues es donde desempeña las actividades de mantenimiento del hogar y donde satisface las necesidades afectivas de sus integrantes (Ariza y de Oliveira, 1997). Esta percepción social moldea, a su vez, las relaciones de pareja, promoviendo los estereotipos de hombre-fuerte-proveedor y mujer-tierna-cuidadora (Cantera y Blanch, 2010).

En el presente trabajo no se asume que los estereotipos en razón de género sean un fenómeno social espontáneo, inevitable e inofensivo, por el contrario, la motivación para su análisis radica en que se les reconoce como expresiones inducidas por un sistema de dominación masculina que lesionan aspectos sociales, personales, económicos, culturales y políticos de la vida de las personas, basándose en ficciones y realidades intersubjetivas respecto a las características de ambos sexos.

El sistema patriarcal ha sido históricamente sustentado en mitos que trascienden las diferencias biológicas entre hombres y mujeres. Si bien la jerarquía del género ha sido la de mayor importancia en todas las sociedades humanas, "la mayoría de las leyes, normas, derechos y obligaciones que definen la masculinidad o la feminidad reflejan más la imaginación humana que la realidad biológica” (Harari, 2019, pp. 169-170). Dicha jerarquía se ha configurado tan injusta como imaginaria, pues ha privilegiado artificialmente la posición social de los hombres frente a las 
mujeres en todos los ámbitos de la vida pública y privada, conformando y extendiendo una realidad explícitamente sexista.

El sexismo se define como un conjunto de expresiones individuales (actitudes, creencias y comportamientos) o prácticas grupales (organizacionales, institucionales y culturales), basadas en evaluaciones negativas de las personas a partir de su sexo, o que promueven la desigualdad entre hombres y mujeres (Swim \& Hyers, 2009).

En este trabajo los estereotipos bajo estudio se consideran sexistas porque no solamente postulan las diferencias entre hombres y mujeres respecto a sus rasgos de personalidad, habilidades, ocupaciones, intereses, apariencia física o expresión de sus emociones, sino que además promueven un trato diferenciado hacia hombres y mujeres a partir de generalizaciones y simplificaciones absurdas, que conducen a la normalización de las desigualdades de género en la sociedad (Swim \& Hyers, 2009), desigualdades que, en el caso de México, se presentan en áreas críticas como la educativa, laboral, participación política o emprendimiento (Camarena et al, 2015).

Así, todo estereotipo de género es un estereotipo sexista en la medida en que justifica el statu quo patriarcal, e instituye descripciones ficticias como prescripciones sociales opresoras para las mujeres.

\section{Factores determinantes de los estereotipos \\ sexistas}

Los antecedentes presentes en la literatura proponen un conjunto de variables relevantes para el análisis explicativo de la incidencia de estereotipos sexistas en una población. Concretamente, se han encontrado premisas teóricas y evidencia empírica que destacan el papel del sexo, la edad, la escolaridad, el nivel de ingreso, la conformación familiar, el estado civil y la adscripción étnica como factores determinantes de la presencia de estos estereotipos en las personas.

Diversos estudios han comprobado una mayor incidencia de estereotipos sexistas en hombres que en mujeres (Cubillas et al, 2016; García y de Oliveira, 2005; Hernández y González, 2016; Salguero et al, 2016), un resultado predecible en la medida en que los beneficios del orden social patriarcal recaen justamente en ellos.

En cuanto a la edad, también es claro que las personas con mayor incidencia de estereotipos sexistas suelen ser más grandes (García y de Oliveira, 2005), marcando una diferencia congruente con el entorno social que le ha tocado vivir a cada generación, y que limita la asimilación de ideales igualitarios en las personas de mayor edad, mientras que los jóvenes juegan un papel activo en la modernización de los roles de cada sexo (Rocha-Sánchez y Díaz-Loving, 2005).

Dado que los estereotipos sexistas son construcciones sociales cognitivas, la educación juega un papel fundamental en su incidencia entre los individuos. Al 
respecto, se asume que a mayor educación aumenta la capacidad de reflexión de las personas respecto a la revaloración de la posición de hombres y mujeres en el ámbito familiar y profesional (Rocha-Sánchez y Díaz-Loving, 2005). Por otro lado, el que un mejor nivel educativo se correlacione con mejores oportunidades de ingreso conduce a que sectores marginados tengan mayor incidencia de estereotipos sexistas, y que conforme mejora la clase social aumente la contradicción de los estereotipos tradicionales (Stern, 2007).

La conformación familiar, y en particular la figura de jefe o cabeza de familia, puede incidir también en los estereotipos sexistas, ya que determinados arreglos familiares abren espacios de flexibilización de las estructuras de autoridad tradicionales, y específicamente en hogares matrifocales se ha observado una valoración especial del papel de la mujer como sostén económico (Ariza y de Oliveira, 1997). Además, se ha encontrado que familias con jefatura femenina cuentan con mayores tasas de participación de la mujer en el mercado laboral (Acosta, 2001), así como relaciones más igualitarias y solidarias entre sus miembros (García y de Oliveira, 2005).

Respecto al estado civil, Rojas (2016) propone que los roles de género tradicionales son alimentados por esquemas patriarcales y religiosos de la vida conyugal, en tanto que la modernización social los cuestiona y promueve el replanteamiento del matrimonio como forma de vida. Así, las disoluciones conyugales podrían conducir a una mayor autonomía de las mujeres y la confrontación de estereotipos (García y de Oliveira, 2005).

Finalmente, la intersección de las categorías género y etnicidad ha sido abordada de manera incipiente, en particular desde el campo de los estereotipos. Autoras como Poeschel-Renz (2003) afirman que la construcción de una identidad de género involucra la articulación de elementos como imágenes y símbolos culturales, que a su vez derivan de prácticas y discursos sociales que configuran las relaciones de género dentro y fuera del ámbito cultural de personas con un sentido de pertenencia étnica. Rosales (2010, p. 42) postula, en el mismo sentido, que la construcción de la identidad de género en personas indígenas está determinada por su grupo étnico, a través de los aspectos sociales, históricos y culturales que forjan las expectativas sobre lo masculino y lo femenino.

También, respecto a la adscripción étnica, cobra relevancia el análisis de personas afrodescendientes, quienes cuentan con una presencia importante en el estado de Guerrero. La intersección de las categorías raza y sexo/género ha contado con reflexiones desde la afrodescendencia o negritud, que demuestran un impacto diferenciado en quienes se ven atravesados de manera conjunta tanto por el sexismo como por el racismo (Curiel, 2007), siendo comúnmente hipersexualizados mediante 
estereotipos acerca de su deseo y disponibilidad sexual (Hellebrandová, 2014; Alcocer, 2019).

\section{Metodología}

Se utilizan datos de la Encuesta de Seguridad Ciudadana en Acapulco (ESCA) del año 2019, en la cual se realizaron entrevistas cerradas a hombres y mujeres mayores de edad en sus viviendas, en siete colonias o barrios del área urbana del municipio de Acapulco. Esta encuesta recopila información sobre incidencia delictiva y percepción de inseguridad, pero también incluye información sociodemográfica de las personas encuestadas y una batería de preguntas sobre estereotipos sexistas, variables de interés para el presente trabajo. Utiliza un muestreo aleatorio simple sobre el total de viviendas habitadas $(\mathrm{N}=24,598)$, con una proporción $\mathrm{p}=13.7 \%$, un nivel de confianza del $95 \%$ y un error de estimación de $1.5 \%$, con un tamaño de muestra resultante de $\mathrm{n}=1,876$ encuestas, e incorporando la aleatoriedad de los participantes mediante saltos sistemáticos en las viviendas encuestadas ${ }^{2}$.

Las variables sociales retomadas de la encuesta, así como su construcción, se presentan en la tabla 1.

Tabla 1. Descripción de variables sociales

\begin{tabular}{lll}
\hline Variable & Tipo & Valores \\
\hline Sexo del encuestado & Dicotómica & 1: Hombre \\
& & $0:$ Mujer
\end{tabular}

Edad

Numérica

Años de edad cumplidos

al momento de la

encuesta

Escolaridad

Ordinal

1: Sin escolaridad

2: Primaria

3: Secundaria

4: Preparatoria

5: Profesional o posgrado

Hogar con jefa de familia Dicotómica 1: Sí

\footnotetext{
${ }^{2}$ Los datos completos de la ESCA 2019 se encuentran disponibles para su consulta en la siguiente dirección: https://doi.org/10.5281/zenodo.4338682
} 
0: No

$\begin{array}{ll}\text { Ingreso mensual per } & \text { Ordinal } \$ 600 \text { o menos } \\ \text { cápita } & 2: \text { de } \$ 601 \text { a } \$ 1,200 \\ & 3: \text { de } \$ 1,201 \text { a } \$ 1,800 \\ & 4: \text { de } \$ 1,801 \text { a } \$ 2,400 \\ & 5: \text { de } \$ 2,401 \text { a } \$ 3,000 \\ 6: \$ 3,001 \text { o más }\end{array}$

Estado civil

Dicotómicas

1: Sí

Soltero

0: No

Casado

Unión libre

Divorciado

Separado

Viudo

Auto adscripción

Dicotómicas

1: Sí

Indígena

$0:$ No

Afrodescendiente

Fuente: elaboración propia. Cifras monetarias en pesos mexicanos (MXN).

Respecto a los estereotipos sexistas, en la encuesta se cuestionó a las personas de la siguiente manera: de acuerdo con lo que usted piensa, responda sí o no a las siguientes preguntas. ¿Usted cree que...

1. ... los hombres deben ganar más salario que las mujeres?

2. ... los hombres deben ocupar mejores puestos que las mujeres en los trabajos?

3. ... las mujeres que trabajan descuidan a sus hijos

4. ... las mujeres casadas deben tener relaciones sexuales con su esposo cuando él quiera?

5. ... las mujeres deben vestirse sin escotes para que no las molesten los hombres?

6. ... sólo las mujeres deben ser las responsables del cuidado de los hijos?

7. ... la naturaleza de la mujer la inclina a realizar las tareas domésticas? 
Con las respuestas a los reactivos anteriores se generaron siete variables dicotómicas que toman el valor de uno cuando el encuestado responde estar de acuerdo con la afirmación, y toman el valor de cero cuando no están de acuerdo, con la intención de analizar el efecto de las variables sociales consideradas en el estudio sobre la probabilidad de que una persona esté de acuerdo con el estereotipo en cuestión.

La relación funcional propuesta es que la probabilidad de estar de acuerdo con cada estereotipo sexista está determinada por los siguientes atributos sociales: sexo, edad, escolaridad, ingreso per cápita del hogar, hogar con mujer jefa de familia, estado civil y auto adscripción étnica. El modelo empírico a estimar, entonces, corresponde a una variable dependiente dicotómica, de la cual se pretende calcular la probabilidad de respuesta con valor uno, dado un vector de variables explicativas, de las cuales se obtendrá su efecto marginal individual según la lógica de un modelo de regresión logística (Wooldridge, 2015, pp. 584-593).

\section{Ecuación 1. Modelo empírico}

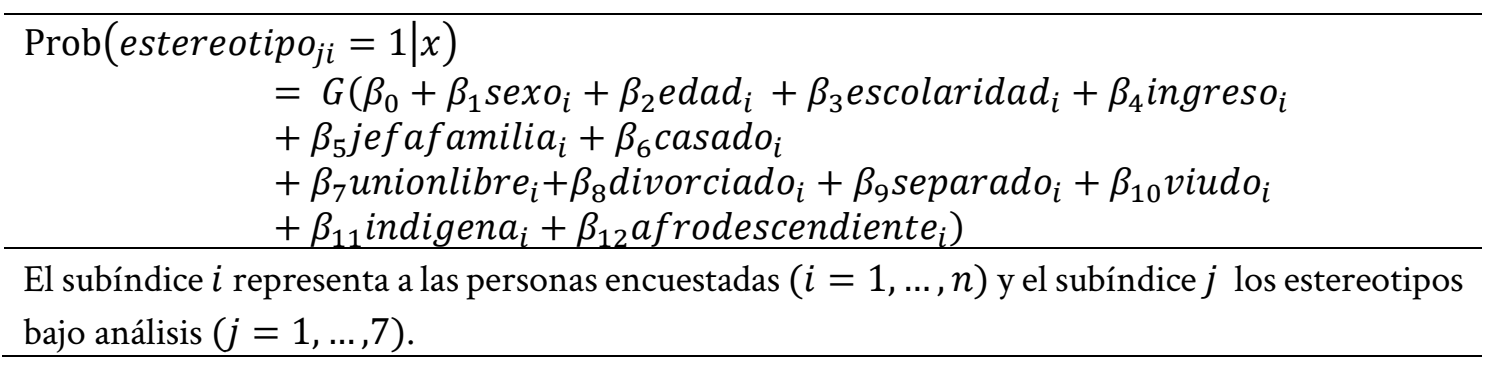

En la ecuación previa, $G$ corresponde a la función de distribución logística con valores entre cero y uno. La estimación de una regresión tipo logit mediante el método de máxima verosimilitud permite encontrar el efecto de cada variable independiente sobre el logaritmo del momio $p /(1-p)$, donde $p$ es la probabilidad de que la variable dependiente tome el valor de uno, dado el vector de variables independientes del modelo, sin la necesidad de suponer una relación lineal ni la normalidad u homocedasticidad de dichas variables (Garson, 2014, p. 10).

Así, al estimar siete regresiones logísticas, una para cada estereotipo sexista bajo análisis, se podrá calcular el efecto marginal de cada variable social sobre la probabilidad de estar de acuerdo con el estereotipo en cuestión. Considerando la naturaleza teórica del fenómeno bajo estudio, así como los antecedentes empíricos encontrados en la literatura, se proponen las siguientes hipótesis:

1. La probabilidad de estar de acuerdo con estereotipos sexistas es mayor en hombres que en mujeres. 
2. A mayor edad, mayor probabilidad de estar de acuerdo con estereotipos sexistas.

3. A mayor escolaridad, menor probabilidad de estar de acuerdo con estereotipos sexistas.

4. A mayor nivel de ingreso, menor probabilidad de estar de acuerdo con estereotipos sexistas.

5. La probabilidad de estar de acuerdo con estereotipos sexistas es menor en personas que viven en hogares donde una mujer es jefa de familia.

6. La probabilidad de estar de acuerdo con estereotipos sexistas es menor en personas con alguna disolución conyugal -divorciadas o separadas-.

7. La probabilidad de estar de acuerdo con estereotipos sexistas es mayor en personas con alguna auto adscripción étnica -indígenas o afrodescendientes-.

En general, se espera que las hipótesis anteriores describan el comportamiento de las variables sociales en todas las regresiones, a pesar de que algunos efectos pudieran tener mayor impacto en ciertos estereotipos. Por ejemplo, en aquellos hogares con una mujer jefa de familia podría esperarse una relación explicativa particularmente importante en los estereotipos relativos al salario y posición de la mujer en el mercado laboral; o bien, el estado conyugal podría incidir con mayor posibilidad en los estereotipos referentes al cuidado de los hijos y el desempeño de actividades domésticas.

\section{Resultados}

El uso de datos provenientes de un muestreo probabilístico aleatorio en un estudio sobre estereotipos sexistas no solamente proporciona un tamaño muestral pertinente para el manejo de métodos explicativos multivariantes, como la regresión logística, sino que además constituye un conjunto de información con perfiles sociodemográficos diversos que enriquecen el análisis del fenómeno ${ }^{3}$.

La muestra total del estudio incluyó personas de 18 a 89 años, con una edad promedio de 47 años. La distribución por sexo fue de $44 \%$ hombres y $56 \%$ mujeres. En términos de escolaridad, el 10\% reveló no tener ninguna, 20\% primaria, 23\%

\footnotetext{
${ }^{3}$ Existen otras fuentes secundarias que permiten el análisis de estereotipos sexistas con mayor cobertura geográfica en México, pero que no presentan esta diversidad de perfiles sociodemográficos. Por ejemplo, la Encuesta Nacional sobre la Dinámica de las Relaciones en los Hogares (ENDIREH) recoge información en todo el país sobre la opinión de roles masculinos y femeninos similares a los estereotipos analizados en el presente trabajo, pero solamente encuesta a mujeres mayores de 15 años.
} 
secundaria, $26 \%$ preparatoria y $21 \%$ profesional o posgrado. El estado civil más frecuente fue casado con $42 \%$, seguido de soltero con $28 \%$, unión libre con $12 \%$, viudo con $10 \%$, separado con $5 \%$ y divorciado con $3 \%$. En cuanto al ingreso mensual aproximado, prevalecen niveles muy bajos, pues prácticamente la mitad de la muestra tiene un ingreso mensual per cápita estimado de \$1,200 MXN o menos ${ }^{4}$. En una de cada cinco viviendas encuestadas se contaba con una mujer como jefa de familia. Finalmente, la presencia de indígenas y afrodescendientes fue de alrededor de $28 \%$ y $14 \%$, respectivamente.

Respecto a la incidencia de los siete estereotipos sexistas de interés para el presente estudio, aquel que tuvo una mayor aceptación entre las personas encuestadas fue que las mujeres que trabajan descuidan a sus hijos, pues el 53\% estuvo de acuerdo con dicho enunciado. Otros estereotipos frecuentes fueron que la naturaleza de la mujer la inclina a realizar las tareas domésticas (30\%) y que las mujeres deben vestirse sin escotes para que no las molesten los hombres (29\%). Así, los estereotipos que suscribe en mayor medida la muestra encuestada versan sobre la condición doméstica de la mujer, tanto en quehaceres del hogar como en la crianza materna, así como en el recato necesario en su manera de vestir por motivos de hostilidad heterosexual (Expósito et al, 1998). Por otro lado, los estereotipos con menor presencia fueron que sólo las mujeres deben ser responsables del cuidado de los hijos (18\%), que los hombres deben ganar más salario que las mujeres (13\%), que las mujeres casadas deben tener relaciones sexuales con su esposo cuando él quiera (13\%), y que los hombres deben ocupar mejores puestos que las mujeres en los trabajos (11\%).

Otra manera de revisar la presencia de estereotipos sexistas es identificar también su intensidad, es decir la cantidad de estereotipos que suscribe una persona encuestada. En este sentido, cabe destacar que cerca del 31\% de la muestra no suscribió ninguno de los siete estereotipos; el 28\% estuvo de acuerdo solamente con uno, y un $17 \%$ estuvo de acuerdo con dos de ellos; finalmente, un $24 \%$ de la muestra estuvo de acuerdo con tres o más estereotipos.

El siguiente paso es revisar el efecto de cada variable social sobre la probabilidad de que las personas encuestadas estén de acuerdo con cada uno de los estereotipos bajo análisis. Para tal efecto, se estimaron las regresiones logísticas de cada estereotipo, para posteriormente calcular el efecto marginal de cada atributo sociodemográfico sobre dicha probabilidad. Los resultados de las siete estimaciones se muestran en la tabla 2. De acuerdo con el estadístico F, los modelos estimados cuentan con significancia estadística global y, aunque la bondad de ajuste de los

\footnotetext{
${ }^{4}$ Este indicador se calcula de manera indirecta, pues la encuesta pregunta por el ingreso mensual total aproximado del hogar si se sumara el ingreso de todos los habitantes de la vivienda, y en otro reactivo se pregunta por el total de sus habitantes, incluyendo infantes y personas de la tercera edad.
} 
modelos es relativamente baja según el criterio de la pseudo- $\mathrm{R}$ cuadrada, el porcentaje de casos correctamente clasificados por los modelos va de un 71 hasta un $91 \%$.

Tabla 2. Resultados de las regresiones logísticas

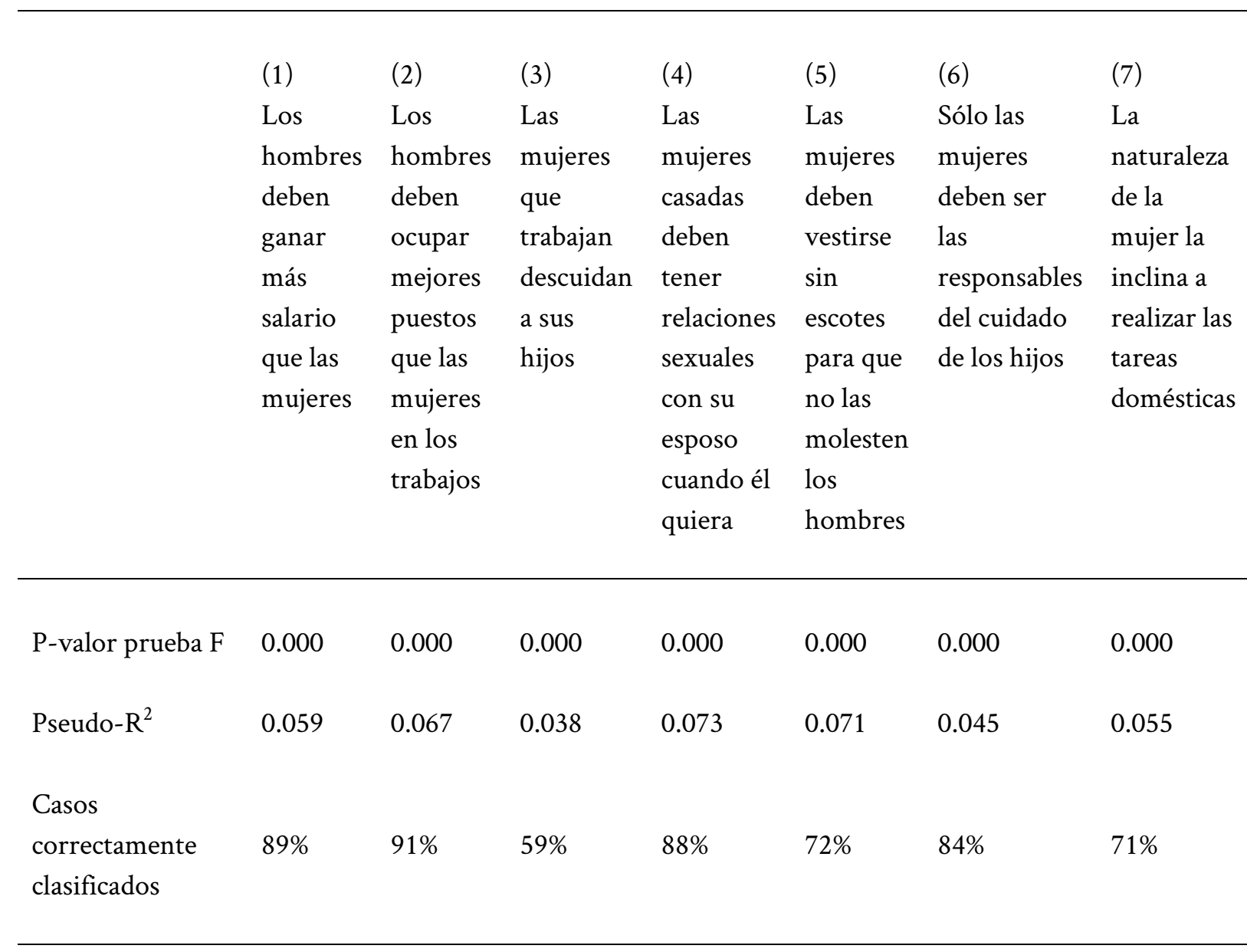

Efectos marginales

$\begin{array}{llllllll}\begin{array}{l}\text { Sexo (base: } \\ \text { hombres) }\end{array} & 0.036^{* *} & 0.031^{* *} & 0.058 & 0.036^{* *} & 0.052 & 0.017 & -0.007 \\ \text { Edad } & 0.001 & -0.001 & 0.003^{* * *} & 0.002^{* * *} & 0.004^{* * *} & 0.002^{* *} & 0.004^{* * *} \\ & - & - & -0.049^{* * *} & -0.024^{* * *} & - & -0.031^{* * *} & -0.038^{* * *} \\ \text { Escolaridad } & -0.023^{* * *} & 0.025^{* * *} & & & 0.040^{* * *} & & \\ & -0.007 & -0.010^{* *} & -0.025^{* * *} & -0.007 & -0.011 & -0.009 & -0.010 \\ \begin{array}{l}\text { Ingreso } \\ \text { Hogar con jefa de }\end{array} & 0.026 & 0.036 & 0.000 & 0.007 & 0.017 & 0.020 & 0.050 \\ \text { familia } & & & & & & & \end{array}$


Estado civil

(base: solteros)

$\begin{array}{rrrrrrrr}\text { Casado } & 0.005 & -0.016 & 0.009 & -0.035 & 0.029 & -0.065^{* * *} & -0.041 \\ \text { Unión libre } & -0.030 & - & 0.083 & -0.041 & -0.018 & -0.051 & -0.067 \\ & & 0.051^{* * *} & & & & & \\ \text { Divorciado } & 0.043 & 0.068 & 0.061 & -0.013 & -0.014 & 0.107 & -0.059 \\ \text { Separado } & -0.020 & -0.011 & 0.030 & -0.013 & 0.010 & -0.065^{* *} & -0.018 \\ \text { Viudo } & 0.039 & 0.052 & -0.019 & 0.020 & 0.033 & -0.047 & -0.024\end{array}$

Auto adscripción

étnica

$\begin{array}{rccccccc}\text { Indígena } & 0.017 & 0.013 & 0.064 & -0.012 & 0.047 & -0.003 & 0.042 \\ \text { Afrodescendiente } & 0.042 & 0.015 & 0.053 & 0.031 & 0.073 & 0.033 & 0.096^{* *}\end{array}$

Fuente: elaboración propia. *** Significativo al 1\%; ** significativo al 5\%.

Los efectos marginales encontrados, así como su significancia estadística, permiten contrastar las hipótesis propuestas en la sección anterior. En lo que concierne al sexo de las personas encuestadas, la evidencia indica que los hombres tienen mayor probabilidad -entre 3.1 y 3.6\%- de estar de acuerdo en tres de los estereotipos bajo análisis: que los hombres deben ganar un salario mayor, ocupar mejores puestos en los trabajos, y que las mujeres deben tener relaciones cuando el esposo quiera; en los cuatro estereotipos restantes, no hay diferencia estadísticamente significativa entre hombres y mujeres.

Sobre la hipótesis de que a mayor edad la probabilidad de estar de acuerdo con estereotipos sexistas aumenta, los resultados indican que así ocurre en todos los estereotipos, con excepción de los dos relativos al salario y puestos de la mujer en el mercado laboral. La magnitud de este efecto ronda entre 0.2 y $0.4 \%$ por cada año alrededor de la edad promedio. Así, suponiendo que el efecto marginal de la edad se comporta de manera lineal ${ }^{5}$, una persona de 67 años tiene $4 \%$ mayor probabilidad de

\footnotetext{
${ }^{5}$ La alternativa es que el efecto marginal de la edad se comporte de manera cuadrática. Sin embargo, al introducir dicha variable elevada al cuadrado, no resultó estadísticamente significativa en ninguno de los modelos.
} 
estar de acuerdo con que sólo las mujeres son responsables del cuidado de los hijos, en comparación con una persona de 47 años; o bien, una persona de 47 años tiene $8 \%$ mayor probabilidad de estar de acuerdo con que las mujeres deben vestirse sin escotes para que no las molesten los hombres, en comparación con una persona de 27 años ${ }^{6}$.

La hipótesis de que la escolaridad reduce la probabilidad de estar de acuerdo con estereotipos sexistas es la única que encontró evidencia a favor en todos los modelos. Así, se puede afirmar de manera contundente que la escolaridad de las personas es uno de los atributos sociales más importantes al momento de predecir la prevalencia de dichos estereotipos. En cuanto a la magnitud del efecto, el impacto de tener preparatoria respecto a quienes solamente tienen secundaria, por ejemplo, reduce la probabilidad de estar de acuerdo con algún estereotipo sexista desde un 2.3 y hasta 4.9\%, dependiendo del estereotipo en cuestión.

El ingreso no tuvo efectos marginales significativos salvo en dos estereotipos: el relativo a que los hombres deben ocupar mejores puestos que las mujeres, y que las mujeres que trabajan descuidan a sus hijos. En estos dos casos el signo del efecto fue, como se esperaba, negativo, aunque la falta de significancia estadística en el resto de los estereotipos sugiere que la relación entre el nivel de ingreso y la prevalencia de estereotipos sexistas no es tan generalizada como con la edad o la escolaridad.

Contrario a lo que se esperaba, el que el hogar tenga una mujer como jefa de familia no resultó tener un efecto estadísticamente significativo sobre la probabilidad de estar de acuerdo con algún estereotipo sexista, ni siquiera con aquellos relativos a la posición jerárquica y salarial de la mujer en el mercado laboral.

Tampoco el estado civil tuvo un efecto sobre los estereotipos sexistas como el que se había propuesto en las hipótesis. En general, la probabilidad de estar de acuerdo con algún estereotipo sexista no difiere entre personas solteras de otros estados civiles, salvo en dos casos particulares. En el primero, las personas casadas o separadas tienen 6.5\% menor probabilidad de estar de acuerdo con que sólo la mujer debe ser responsable del cuidado de los hijos, comparadas con una persona soltera. En otras palabras, el matrimonio -vigente o sin disolución legal- pudiera constituir un estado civil que menoscaba la idea de exclusividad de la crianza de los hijos en la figura materna. En el segundo caso, las personas en unión libre tienen 5.1\% menor probabilidad de estar de acuerdo con que los hombres deben ocupar mejores puestos que las mujeres en los trabajos, respecto a los demás estados civiles. Este resultado

\footnotetext{
${ }^{6}$ Las diferencias de probabilidad entre personas con 20 años de separación se presentan con fines ilustrativos. En realidad, el efecto marginal de la edad se calcula alrededor de la media y solamente son válidos a un año de distancia, es decir, de 46 a 47 años, o bien de 47 a 48. En términos estadísticos, no hay garantía de que el efecto marginal se mantenga constante a lo largo de todo el rango de edad, aun suponiendo que el efecto de dicha variable es lineal.
} 
pudiera sugerir que la posición jerárquica de la mujer en el mercado laboral es particularmente importante en este tipo de sociedades conyugales.

Finalmente, la auto adscripción étnica como indígena o afrodescendiente tampoco tuvo efectos significativos sobre la prevalencia de estereotipos, salvo en un caso. Resulta que las personas que se identifican como afrodescendientes, tienen $9.6 \%$ mayor probabilidad de suscribir el estereotipo de que la naturaleza de la mujer la inclina a realizar las tareas domésticas. Este peculiar resultado, más que considerarse como una excepción digna de soslayar, podría motivar estudios futuros que profundicen en la construcción de ese estereotipo en personas o comunidades afrodescendientes, ya que la magnitud porcentual de dicho impacto destaca del resto de efectos marginales obtenidos en las estimaciones.

Además de los efectos marginales individuales de las características sociodemográficas en cada uno de los modelos, es posible explorar algunos efectos conjuntos mediante la interacción de variables que hayan resultado significativas para explicar un determinado estereotipo. Por ejemplo, el efecto marginal conjunto entre escolaridad y sexo, revela que pensar que "los hombres deben ganar más salario que las mujeres" (Gráfica 1) es un estereotipo que suscriben más los varones, independientemente del nivel de escolaridad. Aunque la brecha se acorta conforme se avanza en los niveles educativos, esta situación se replica en el estereotipo de que "los hombres deben ocupar mejores puestos que las mujeres en los trabajos". En el estereotipo referente a que "las mujeres casadas deben tener relaciones sexuales con su esposo cuando él quiera" (Gráfica 2), por el contrario, se aprecia un efecto marginal creciente entre la edad y el sexo, en el cual la brecha entre hombres y mujeres se incrementa conforme aumenta la edad. 
Gráfica 1. Estereotipo “los hombres deben ganar más salario que las mujeres"

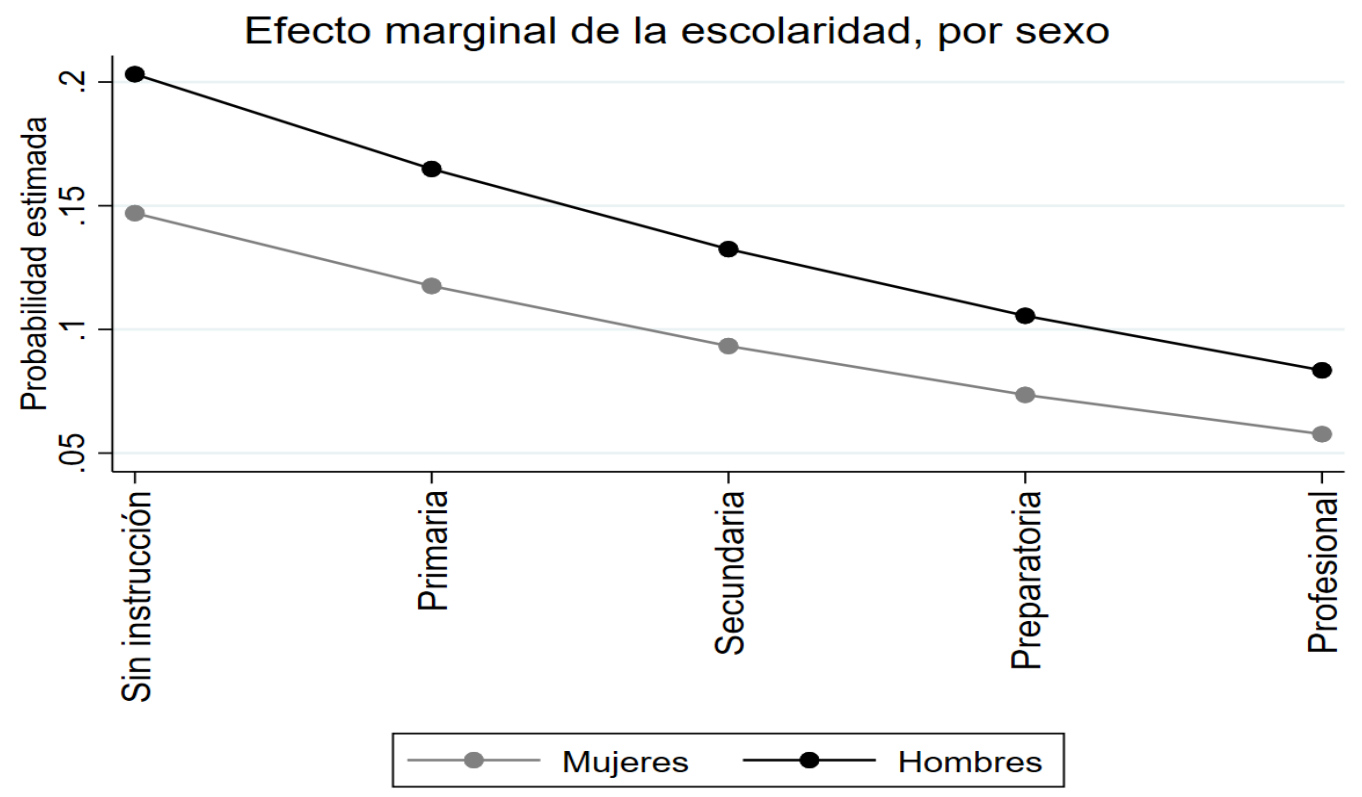

Fuente: elaboración propia.

Gráfica 2. Estereotipo "las mujeres casadas deben tener relaciones sexuales con su esposo cuando él quiera"

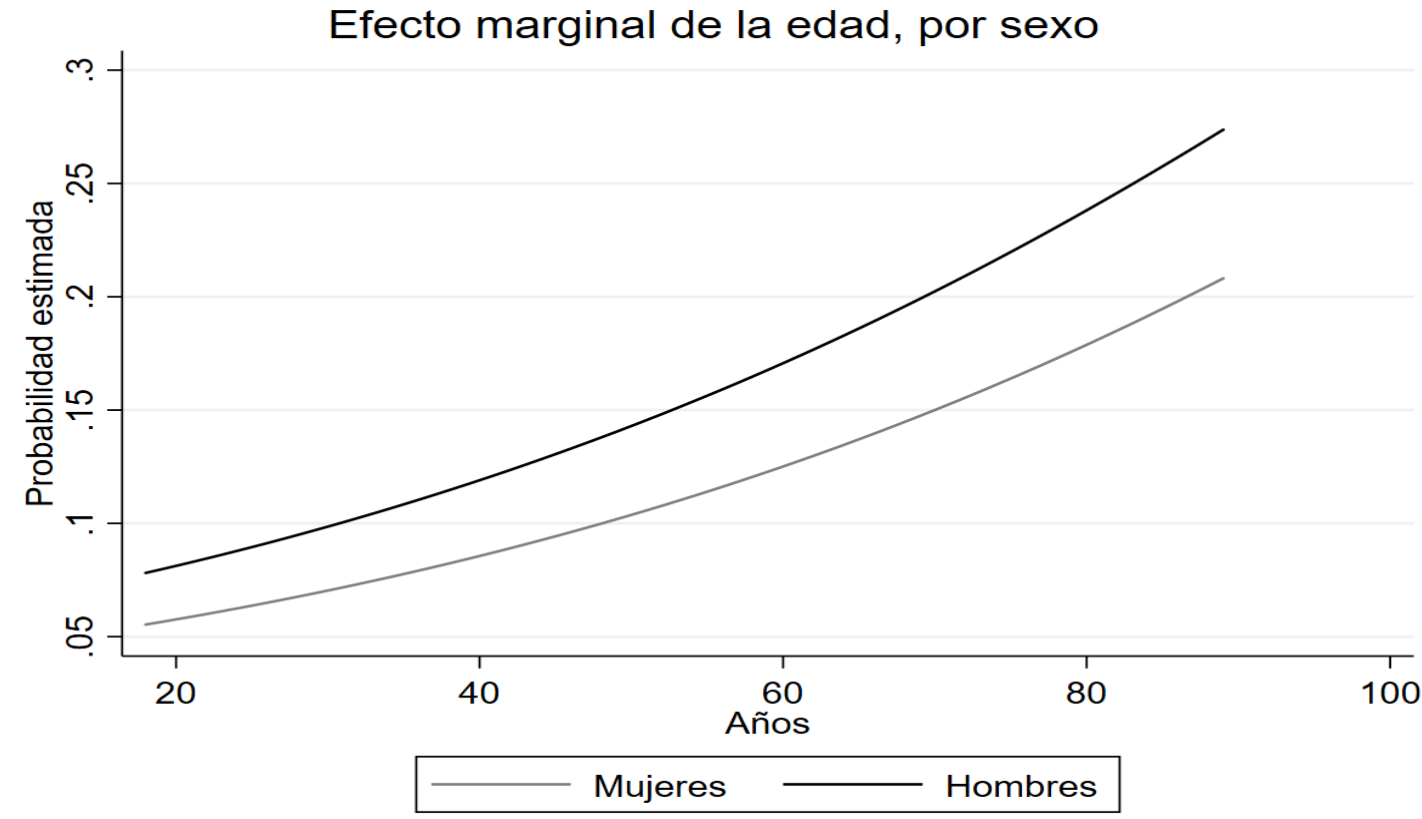

Fuente: elaboración propia.

Hasta aquí, los hallazgos empíricos conducen a establecer que la edad y la escolaridad, en términos generales, constituyen los factores explicativos más importantes de la prevalencia de estereotipos sexistas. Otros atributos sociales tienen 
un impacto específico en ciertos estereotipos, como el caso del estado civil sobre la creencia de que el cuidado de los hijos es una tarea exclusiva de las mujeres, o la auto adscripción como afrodescendiente sobre la propensión de las personas a creer que la naturaleza de la mujer la inclina a las labores domésticas. Sin embargo, un resultado que no queda del todo claro en términos explicativos, es el papel del sexo: ¿por qué es relevante en algunos estereotipos y en otros no? Siendo que el fenómeno de estudio constituye una expresión de sexismo, ¿no debería ser el sexo un elemento clave para determinar la prevalencia de todos los estereotipos bajo análisis?

Con el fin de esclarecer la controversia se indagaron diversas explicaciones. Una de ellas era que el efecto explicativo del sexo podría haber sido capturado por algún otro atributo, como la edad. Esto podría tener sentido ya que en los cuatro estereotipos en los cuales el sexo no fue significativo, la edad sí lo fue, mientras que en los dos estereotipos donde la edad no fue significativa, el sexo sí lo fue. Lo anterior podría ocurrir si algún grupo de edad tuviera una presencia atípica de hombres o de mujeres, es decir, si alguno de los sexos estuviera sobrerrepresentado en una determinada edad. Este no fue el caso, ya que al comprobar la distribución de la edad en hombres y mujeres por separado, no se encontraron diferencias sustanciales.

Otra posibilidad implicaba la intervención de variables fuera del modelo explicativo, que pudieran interactuar con el sexo de la persona encuestada provocando la intermitencia de su significancia estadística entre los estereotipos. Una relación que despertó un interés particular fue ¿cómo podría afectar el sexo de la persona que lleva a cabo la entrevista a las respuestas de un sexo en particular? En otras palabras, la probabilidad de que un hombre o una mujer suscriban un determinado estereotipo sexista, ¿depende de si quien pregunta es un encuestador o una encuestadora? Esta alternativa adquirió relevancia no solamente porque podría explicar la controversia analítica del papel del sexo en la prevalencia de dichos estereotipos, sino también por las implicaciones metodológicas para el diseño de encuestas en materia de estereotipos, prejuicios o comportamientos sexistas.

El efecto del sexo del encuestador ya ha sido analizado en otras investigaciones mediante encuestas y estudios de opinión que abordan temas de género, documentando una mayor sensibilidad a influir las respuestas de hombres que de mujeres, así como una mayor probabilidad de presentar sesgos en datos locales comparados con datos nacionales (Flores-Macías y Lawson, 2008). Otros estudios, en cambio, han documentado un efecto similar sobre hombres y mujeres, quienes exhiben un sesgo en las respuestas no solamente a las preguntas relacionadas con el género, sino también en preguntas neutras y hasta en las sociodemográficas (Verge, 2009). 
Para comprobar el efecto del sexo de la persona que realiza la encuesta, se procedió a calcular las regresiones logísticas de los siete estereotipos de manera separada para hombres y mujeres, eliminando la variable que capturaba el sexo de la persona a quien se entrevistaba e incorporando el sexo de quien realizaba la entrevista. Cabe mencionar que las cuadrillas de trabajo de campo para el proyecto estadístico de donde provienen los datos estaban conformadas mayoritariamente por mujeres, de ahí que solamente el 27\% de las observaciones fueron recopiladas por un hombre.

La intención de estimar los modelos por separado para hombres y mujeres era, en primer lugar, verificar que no cambiaran los efectos marginales y la significancia estadística obtenida en los modelos conjuntos y, en segundo lugar, identificar si el sexo de la persona que realizaba la encuesta tenía un efecto marginal significativo. Al analizar los resultados de las regresiones por sexo, se verificó que los efectos marginales de las variables sociales originalmente contempladas no cambiaban: la edad y la escolaridad continuaban siendo los principales factores explicativos de los siete estereotipos, tanto en hombres como en mujeres.

Respecto al sexo de la persona que realiza la encuesta, se encontraron resultados interesantes (Tabla 3). En las regresiones logísticas de los hombres de la muestra, no se observaron diferencias estadísticamente significativas en la prevalencia de estereotipos sexistas dependiendo de si eran abordados por un encuestador o una encuestadora. En otras palabras, no se encontró evidencia de que los hombres modificaran su opinión respecto a los estereotipos considerados, en función de si le preguntaba un hombre o una mujer.

En las regresiones logísticas de las mujeres de la muestra tampoco se encontraron diferencias estadísticamente significativas en la probabilidad de suscribir los estereotipos sexistas, con excepción de dos casos. Se observó que al preguntarle a una mujer si está de acuerdo con que las mujeres deben vestirse sin escotes para que no las molesten los hombres, o que la naturaleza de la mujer la inclina a realizar las tareas domésticas, si quien realiza la pregunta es un hombre, la probabilidad de estar de acuerdo con alguno de estos estereotipos aumenta un 12\%, en comparación a cuando la pregunta se la hace otra mujer.

Este resultado aumenta la importancia analítica del sexo como variable social explicativa de los estereotipos sexistas, pues el hecho de ser hombre o mujer no solamente interviene en la reflexión individual que llevan a cabo los sujetos para suscribir o no un determinado estereotipo, sino que también incide en cómo un determinado grupo, en este caso las mujeres, revela abiertamente estar de acuerdo con un enunciado sexista, en función de si la pregunta se la hace un hombre o una mujer. El por qué la propensión de las mujeres a revelar su acuerdo con ciertos 
estereotipos sexistas es menor cuando quien pregunta es otra mujer queda para un análisis exhaustivo en estudios futuros. Por ahora basta con destacar dicho hallazgo emergente, con el fin de que sea contemplado en el diseño metodológico de investigaciones orientadas a medir la prevalencia de estereotipos, prejuicios o comportamientos sexistas.

\section{Tabla 3. Efecto del sexo del encuestador}

$\begin{array}{lllllll}(1) & (2) & (3) & (4) & (5) & (6) & (7) \\ \text { Los } & \text { Los } & \text { Las } & \text { Las } & \text { Las } & \text { Sólo las } & \text { La } \\ \text { hombre } & \text { hombre } & \text { mujeres } & \text { mujeres } & \text { mujeres } & \text { mujeres } & \text { naturaleza } \\ \text { s deben } & \text { s deben } & \text { que } & \text { casadas } & \text { deben } & \text { deben ser } & \text { de la } \\ \text { ganar } & \text { ocupar } & \text { trabajan } & \text { deben } & \text { vestirse } & \text { las } & \text { mujer la } \\ \text { más } & \text { mejores } & \text { descuida } & \text { tener } & \text { sin } & \text { responsable } & \text { inclina a } \\ \text { salario } & \text { puestos } & \text { n a sus } & \text { relacione } & \text { escotes } & \text { s del } & \text { realizar } \\ \text { que las } & \text { que las } & \text { hijos } & \text { s sexuales } & \text { para que } & \text { cuidado de } & \text { las tareas } \\ \text { mujeres } & \text { mujeres } & & \text { con su } & \text { nolas } & \text { los hijos } & \text { doméstica } \\ & \text { en los } & & \text { esposo } & \text { moleste } & & \text { s } \\ & \text { trabajos } & & \text { cuando él } & \text { n los } & & \end{array}$

Efecto marginal del sexo del encuestador (base: hombres)

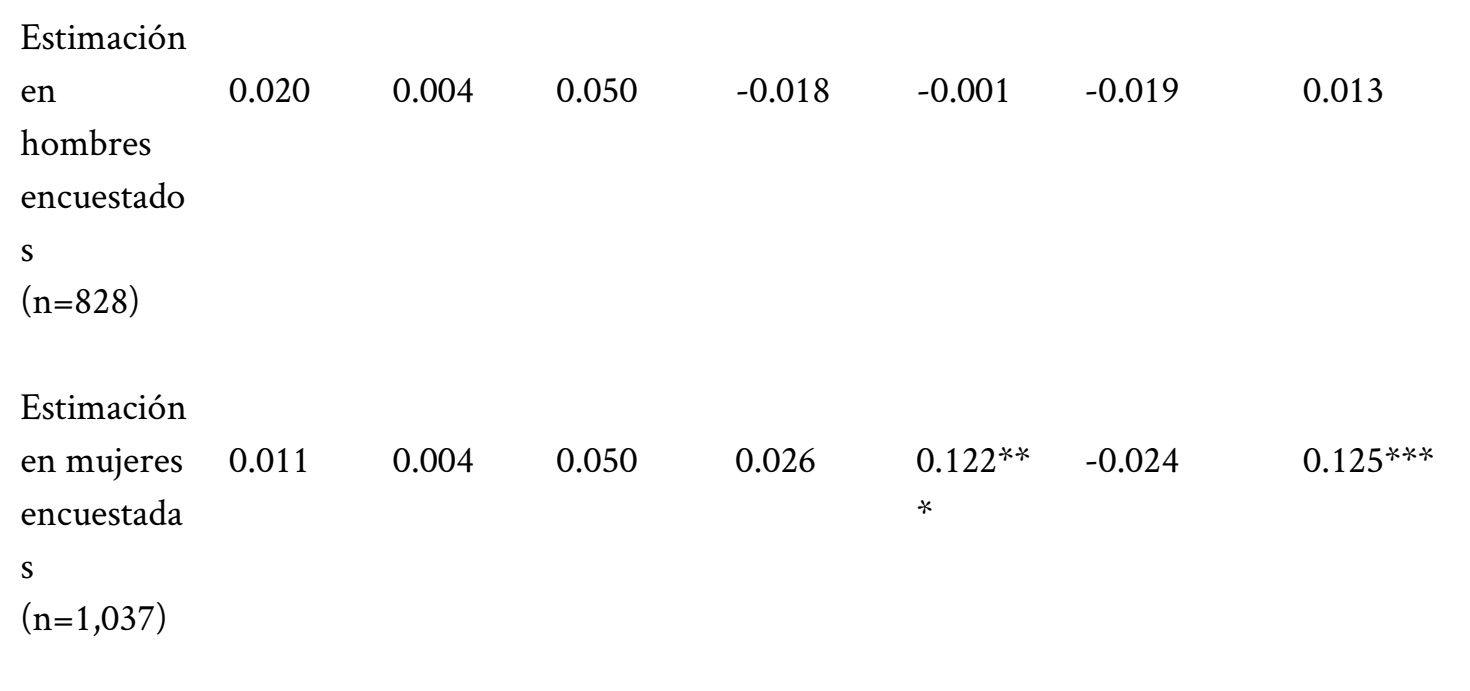

Fuente: elaboración propia. *** Significativo al 1\%. 


\section{Conclusiones}

Este trabajo buscó identificar en términos empíricos los factores que determinan la prevalencia de estereotipos sexistas en las personas. La importancia de dicho objetivo radica en que esos estereotipos normalizan las desigualdades de género en la sociedad y constituyen mecanismos sociales de opresión contra las mujeres. Por tal motivo, la comprensión de las variables sociales que explican la prevalencia de los mismos podría orientar medidas concretas para su mitigación.

Los resultados indican que la prevalencia de estereotipos sexistas aumenta con la edad, ratificando que la asimilación de ideales igualitarios entre hombres y mujeres presenta más resistencia en personas con mayor edad, mientras que los jóvenes aprovechan la modernidad de su entorno social para menguar la prevalencia de estos estereotipos. Así, la brecha generacional se erige como uno de los principales obstáculos para erradicar estas expresiones de sexismo.

Por otro lado, y al ser los estereotipos una construcción social cognitiva, la educación demuestra ser un detonante de la capacidad de reflexión en torno a la revaloración de la posición de la mujer en el ámbito laboral y familiar.

También los factores que no fueron significativos para explicar el fenómeno constituyen hallazgos importantes. Llama la atención que la conformación familiar, concretamente la jefatura femenina en el hogar, no haya tenido un efecto sobre la prevalencia de estereotipos sexistas, y por lo tanto sobre la valoración de la mujer en términos familiares y laborales. En el mismo sentido, la auto adscripción como indígena no tuvo impactos sustanciales sobre la probabilidad de suscribir estereotipos sexistas, mientras que en los afrodescendientes únicamente se observó una predisposición atípica de considerar que la naturaleza de la mujer la inclina a realizar las tareas domésticas.

Un hallazgo emergente del presente análisis es que el sexo de las personas pudiera incidir en la prevalencia de estereotipos sexistas mediante mecanismos que dependen de la interacción con otros sujetos. En términos metodológicos, si bien el sexo de las personas determina la prevalencia de algunos de estos estereotipos, en otros casos es la interacción entre una mujer encuestada y un hombre encuestador la que determina si dicha mujer revelará si suscribe o no el estereotipo en cuestión. El hecho de que las mujeres sean menos propensas a revelar que están de acuerdo con ciertos estereotipos sexistas cuando quien pregunta es otra mujer, es un hallazgo digno de seguimiento en investigaciones futuras por sus implicaciones metodológicas. 


\section{Referencias}

Acosta, F. (2001). Jefatura de hogar femenina y bienestar familiar: resultados de la investigación empírica. Papeles de Población, 7(28), pp. 41-97.

Alcocer, M. (2019). Población afrodescendiente en Guerrero: entre la representación y la apropiación de estereotipos raciales y sexuales. Revista Cultura $y$ Representaciones Sociales, núm. 27, pp. 348-378.

Ariza, M., y de Oliveira, O. (1997). Formación y dinámica familiar en México, Centroamérica y El Caribe. Ibero.Amerikanisches Archiv, 23(1), pp.27-44.

Ashmore, R., del Boca, F., \& Wohlers, A. (1986). Gender stereotypes. En R. Ashmore \& F. del Boca (Eds.), The Social Psychology of Female-Male Relations. A Critical Analysis of Central Concepts (pp. 69-119). Academic Press.

Basow, S. (1992). Gender: Stereotypes and Roles. Thomson Brooks/Cole Publishing Co.

Bonelli, A. (2017). Modalidades vinculares entre padres separados o divorciados e hijos e hijas en la crisis del patriarcado. Journal de Ciencias Sociales, año 5, núm. 8, pp. 99-113.

Camarena, M., Saavedra, M., y Ducloux, D. (2015). Panorama del género en México: Situación actual. Revista Científica Guillermo de Ockham, 13(2), pp. 77-87.

Cantera, L., y Blanch, J. (2010). Percepción social de la violencia en la pareja desde los estereotipos de género. Intervención Psicosocial, 19(2), pp. 121-127.

Castañeda, M. (2007). El machismo invisible regresa. Taurus.

Cook, R., \& Cusack, S. (2010). Gender Stereotyping: Transnational Legal Perspectives. University of Pennsylvania Press.

Cubillas, M., Valdez, E., Domínguez, S., Román, R., Hernández, A., y Zapata, J. (2016). Creencias sobre estereotipos de género de jóvenes universitarios del norte de México. Diversitas: Perspectivas en Psicología, 12(2), pp. 217-230.

Curiel, O. (2007). Los aportes de las afrodescendientes a la teoría y la práctica feminista. Desuniversalizando el sujeto 'mujeres'. En M.L. Femenías, (Comp.), Perfiles del Feminismo Iberoamericano, vol. III (pp. 163-190). Catálogos.

Delgado, G., Novoa, R., y Bustos, O. (1998). Ni tan fuertes ni tan frágiles. Resultados de un estudio sobre estereotipos y sexismo en mensajes publicitarios de televisión $y$ educación a distancia. UNICEF/Programa Nacional de la Mujer. 
Díaz-Cervantes, R. (2014). La perspectiva de género en la comprensión de la masculinidad y la sobrevivencia indígena en México. Agricultura, Sociedad y Desarrollo, 11(3), pp. 359-378.

Ellemers, N. (2018). Gender stereotypes. Annual Review of Psychology, núm. 69, pp. 275-298.

Expósito, F., Moya, M., y Glick, P. (1998). Sexismo ambivalente: medición y correlatos. Revista de Psicología Social, 13(2), pp. 159-169.

Flores-Macias, F., \& Lawson, C. (2008). Effects of interviewer gender on survey responses: findings from a household survey in Mexico. International Journal of Public Opinion Research, 20(1), pp. 100-110.

Frías, S., y Erviti, J. (2012). Patriarcado y estereotipos de género en México: extensión y representación en la imagen. En C. Pinto (Ed.), Otra mirada. Imágenes de identidad en España y México (pp. 183-213). Milrazones.

García, B., y de Oliveira, O. (2005). Las transformaciones de la vida familiar en el México urbano contemporáneo. En X. Valdés y T. Valdés (Coords.), Familia y vida privada. ¿Transformaciones, tensiones, resistencia o nuevos sentidos? (pp. 77106). Facultad Latinoamericana de Ciencias Sociales.

Garson, D. (2014). Logistic Regression: Binary \& Multinomial. Statistical Associates.

Glick, P. \& Fiske, S. (1997). Hostile and benevolent sexism: measuring ambivalent sexist attitudes toward women. Psychology of Women Quarterly, 21(1), pp. 119135.

González, J., y Viadero, M. (2010). Equidad de género y pueblos indígenas desde la perspectiva de la educación para el desarrollo. En Género en la educación para el desarrollo. Abriendo la mirada a la interculturalidad, pueblos indígenas, soberanía alimentaria, educación para la paz (pp. 33-67). Universidad del País Vasco/Instituto de Estudios sobre Desarrollo y Cooperación Internacional/ACSUR-Las Segovias.

Gutmann, M. (2000). Ser hombre de verdad en la Ciudad de México. Ni macho ni mandilón. El Colegio de México.

Harari, Y. (2019). De animales a dioses. Breve historia de la humanidad. Debate.

Hellebrandová, K. (2014). Escapando a los estereotipos (sexuales) racializados: el caso de las personas afrodescendientes de clase media en Bogotá. Revista de Estudios Sociales, núm. 49, pp. 87-100. 
Hernández, A., y González, J, (2016). Los roles y estereotipos de género en los comportamientos sexuales de jóvenes de Coahuila, México: aproximación desde la Teoría Fundamentada. CIENCIA ergo-sum, 23(2), pp. 112-120.

Kite, M., Deaux, K., y Haines, E. (2008). Gender Stereotypes. En F. Denmark, \& M. Paludi (Eds.), Psychology of Women: A Handbook of Issues and Theories (pp. 205236). Praeger.

Lamas, M. (1986). La antropología feminista y la categoría 'género'. Nueva Antropología, 8(30), pp. 173-198.

---- (2002). Cuerpo: diferencia sexual y género. Taurus.

Machillot, D. (2013). Machos y machistas. Historia de los estereotipos mexicanos. Paidós.

Martín, A. (2008). Antropología del género. Culturas, mitos y estereotipos sexuales. Ediciones Cátedra.

Operario, D., \& Fiske, S. (2003). Stereotypes: Content, structures, processes, and context. En R. Brown, \& S. Gaertner (Eds.), Blackwell Handbook of Social Psychology: Intergroup Processes (pp. 22-44). Blackwell Publishing.

Pearson, J., Turner, L., y Todd-Mancillas, W. (1993). Comunicación y género. Paidós.

Pla, I., Adam, A., y Bernabeu, I. (2013). Estereotipos y prejuicios de género: factores determinantes en salud mental. Norte de Salud Mental, 11(46), pp. 20-28.

Poeschel-Renz, U. (2003). Las marcas de la violencia en la construcción sociohistórica de la identidad femenina indígena. Revista Ecuador Debate, núm. 59, pp. 103-122.

Rocha-Sánchez, T., y Díaz-Loving, R. (2005). Cultura de género: La brecha ideológica entre hombres y mujeres. Anales de Psicología, 21(1), pp. 42-49.

Rojas, O. (2016). Mujeres, hombres y vida familiar en México. Persistencia de la inequidad de género anclada en la desigualdad social. Revista Interdisciplinaria de Estudios de Género, 2(3), pp. 73-101.

Rosales, A. (2010). Sexualidades, cuerpo y género en culturas indígenas y rurales. Horizontes Educativos.

Salguero, M., Soriano, M., y Ayala, C. (2016). Estereotipos de género: sexualidad y anticoncepción en jóvenes universitarios de clase media. Investigaciones Feministas, 7(1), pp. 335-352.

Stangor, C. (2009). The Study of Stereotyping, Prejudice, and Discrimination Within Social Psychology. A Quick History of Theory and Research. En T. Nelson 
(Ed.), Handbook of Prejudice, Stereotyping and Discrimination (pp. 1-22). Psychology Press.

Stern, C. (2007). Estereotipos de género, relaciones sexuales y embarazo adolescente en las vidas de jóvenes de diferentes contextos socioculturales en México. Estudios Sociológicos, 25(73), pp. 105-129.

Swim, J., \& Hyers, L. (2009). Sexism. En T. Nelson (Ed.), Handbook of Prejudice, Stereotyping and Discrimination (pp. 407-430). Psychology Press.

Triana, J. (2020). Prevalencia de estereotipos machistas en Acapulco, Guerrero: un estudio correlacional. Revista Ciencia y Mar, 24(71), pp. 35-51.

Verge, T. (2009). Deseabilidad social y género: el efecto del sexo del entrevistador(a) sobre las respuestas del encuestado(a). Metodología de Encuestas, núm. 11, pp. $27-$ 45.

Wooldridge, J. (2015). Introducción a la econometría. Cengage Learning. 